\title{
Correlation of Environment, Nutrition, Genetics, Epigenetics, Microbiota with Neonatal Fetus Development
}

\section{Interrelación Entre Ambiente, Nutrición, Genética, Epigenética, Microbiota y Desarrollo Feto Neonatal}

XVIII International Seminar on Health, Food and Human Nutrition

Corresponding Author:

Iván Enrique Naranjo Logroño

naranjometropolitana@hotmail.com

Published: 9 September 2021

Production and Hosting by

Knowledge E

(a) Iván Enrique Naranjo Logroño et al. This article is distributed under the terms of the Creative Commons Attribution License, which permits unrestricted use and redistribution provided that the original author and source are credited.
Iván Enrique Naranjo Logroño ${ }^{1,2}$, Anthony Alfonso Naranjo Coronel ${ }^{2}$, Angie Daniela Beltrán Vera ${ }^{1}$, and Ashley Carolina Cuzco Macías ${ }^{1}$

${ }^{1}$ Carrera de Medicina, Facultad de Salud Pública, Escuela Superior Politécnica de Chimborazo, Riobamba, Ecuador

${ }^{2}$ COLPOMED Centro Hospital del día, Riobamba, Ecuador

\section{Abstract}

Introduction: From the moment of conception the human being is predisposed to undergo changes in fetal and neonatal development due to various external factors that occur throughout life and can in the long term influence the phenotypic expression of the new being. Objective: The objective of this work is to determine the relationship between the environment, nutrition, genetics, epigenetics and microbiota with neonatal fetus development and how they influence the phenotypic expression of the new being. Methods: A non-systematic search was performed in electronic databases such as COCHRANE, PUBMED, MEDLINE, etc. The bibliographic research was carried out in the period between October 2019 and January 2020. The studies carried out from 2007 to 2019, in the languages of Spanish and English, were included. Results: We found 51 bibliographic sources related to the subject, of which by means of a last simplification, 30 scientific articles were used that provided important information on the subject, and 21 articles were excluded. Conclusion: Research on methods to detect these interactions and to understand the mechanisms of these interactions is just beginning However, there is evidence that they play an important role in human development.

Keywords: neonatal development, epigenetics, microbiota, fetal development, nutrition.

\section{Resumen}

Introducción: Desde el momento de la concepción el ser humano está predispuesto a sufrir cambios en el desarrollo fetal y neonatal debido a diversos factores externos que se presentan a lo largo de la vida y pueden a largo plazo influir en la expresión fenotípica del nuevo ser. Objetivo: El objetivo del presente trabajo es determinar la relación que existe entre el medio ambiente, nutrición, genética, epigenética y microbiota con el desarrollo feto neonatal y como los mismos influyen en la expresión fenotípica del nuevo ser. Métodos: Se realizó una búsqueda no sistemática en bases de datos electrónicas como COCHRANE, PUBMED, MEDLINE, etc. La investigación bibliográfica se realizó en el periodo comprendido entre octubre 2019 y enero 2020. Se incluyeron estudios realizados desde el año 2007 hasta el año 2019, en los idiomas de español e inglés. Resultados: Se encontraron 51 fuentes bibliográficas referentes al tema, de los cuales mediante una última simplificación se utilizaron 30 artículos científicos que aportaron 
información importante del tema y se excluyeron 21 artículos. Conclusiones: La investigación sobre métodos para detectar estas interacciones y para comprender los mecanismos de estas interacciones apenas está comenzando. Sin embargo, hay evidencia de que estas juegan un papel importante en el desarrollo humano.

Palabras Clave: desarrollo neonatal, epigenética, microbiota, desarrollo fetal, nutrición.

\section{Introducción}

A lo largo de la vida, los humanos están expuestos a un sinfín de influencias externas, que empieza desde la etapa prenatal; los efectos de estas exposiciones en dependencia de su impacto pueden provocar cambios en el desarrollo fetal y neonatal, y a largo plazo modificar la expresión fenotípica del nuevo ser, e incluso estos cambios, pueden ser transmitidos a las generaciones futuras; el medio ambiente tiene un profundo efecto en la plasticidad del desarrollo. Se ha especulado que, en respuesta a la homeostasis materna y los estímulos intrauterinos, el feto sufre respuestas predictivas que en teoría resultan en ajustes permanentes de los sistemas homeostáticos, con el objetivo de mejorar la adaptación al ambiente postnatal [1-3].

Está bien establecido que los diferentes entornos prenatales positivos o negativos, pueden cambiar el desarrollo fetal normal y provocar cambios en el nuevo individuo [4]. Por tal motivo esta revisión tiene como objetivo proporcionar una visión completa sobre las complejas interacciones entre ambiente, nutrición, microbiota y sus repercusiones sobre la genética y epigenética, y cómo estas a su vez modifican el desarrollo feto neonatal.

\section{Metodología}

Se realizó una búsqueda no sistemática en bases de datos electrónicas como COCHRANE, PUBMED, MEDLINE, LILACS, SCIENCE DIRECT y SCOPUS. Se tomaron en cuenta artículos en los idiomas de español e inglés. La investigación bibliográfica se realizó en el periodo comprendido entre octubre 2019 y enero 2020.

Como criterios de inclusión se abarcaron ensayos clínicos controlados, randomizados revisiones sistemáticas y metaanálisis en las bases de datos mencionadas anteriormente, bajo la utilización de palabras clave como desarrollo neonatal, epigenética, microbiota, desarrollo fetal y nutrición. Se incluyen estudios realizados desde el año 2007 hasta el año 2019.

\section{Resultados}

Como resultado de la búsqueda, se encontraron 51 fuentes bibliográficas referentes al tema de interrelación entre ambiente, nutrición, genética, epigenética, microbiota y desarrollo feto neonatal, de los cuales mediante una última simplificación considerando 
los criterios de inclusión se utilizaron 30 artículos científicos que aportaron información importante del tema y se excluyeron 21 artículos debido a que no cumplieron con los criterios definidos.

\section{Discusión}

\subsection{Ambiente}

En la actualidad, las investigaciones ratifican que el resultado que conlleva la exposición ambiental en la vida intrauterina es parte fundamental en el desarrollo de diversas alteraciones a futuro. Además, se conoce que la placenta es considerada como el órgano intermediario entre la exposición de los factores ambientales y el impacto que estos infunden en el desarrollo de las aptitudes en los niños $[5,6]$.

\subsection{Estrés}

Como sabemos, el estrés es el conjunto de reacciones fisiológicas presentadas ante un estado de tensión, entre los factores estresantes percibidos por la madre durante el embarazo que pueden influir en la salud de la descendencia se incluyen los estresores físicos, como la desnutrición y toxinas como el alcohol, la nicotina o los bifenilos policlorados; los estresores crónicos psicosociales, como sufrir un trastorno psiquiátrico, cuidar a parientes con enfermedades terminales, o estar expuesto a violencia continua (por ejemplo, violencia doméstica o vivir en una zona de guerra) o pobreza (por ejemplo, hambre o baja estado socioeconómico); estar expuesto a traumas agudos incontrolables graves (desastres naturales, terrorismo, o genocidio), que puede provocar el desarrollo de un trastorno de estrés postraumático (TEPT) [5]

El estrés materno que se relaciona con el ambiente familiar que perjudica a la mujer, es un factor que agrava de manera importante el desarrollo del embarazo. Por otro lado, el maltrato contra la mujer juega un rol destacado en los mecanismos de estrés. Es así que con el paso del tiempo se ha visto que estos niños nacidos de madres expuestas a maltrato tienen una respuesta alterada a procesos de estrés, alteraciones en la regulación del eje hipotálamo- hipófisis-adrenal, con alteraciones en las respuestas autonómicas. Análisis modernos entreveran la asociación entre la depresión materna en etapa prenatal y las alteraciones en el eje hipotálamo-hipófisis-adrenal en niños, hallándose un mecanismo esclareciendo que las alteraciones serían producto de la metilación del gen NR3C1, el cual es receptor de glucocorticoides, sensible al estado emocional de la madre [6].

Cabe acotar que después de la exposición a un estresor agudo, el hipotálamo libera la hormona liberadora de corticotropina $(\mathrm{CRH})$, que promueve la liberación de la hormona adrenocorticotrópica (ACTH) de la glándula pituitaria. Una vez en las glándulas suprarrenales, la ACTH desencadena la liberación de cortisol. Este sistema está autorregulado por una retroalimentación negativa en la que el cortisol inhibe la liberación de $\mathrm{CRH}$ y $\mathrm{ACTH}$ del sistema nervioso central. Sin embargo, durante la exposición al estrés crónico, esta retroalimentación puede verse afectada por el desarrollo de 
resistencia a los glucocorticoides mediada por la desensibilización del receptor de glucocorticoides (GR). Esta situación lleva a una hiperactivación del eje hipotálamopituitario adrenal (HPA) que se ha sugerido para apuntalar el desarrollo de trastornos neuropsiquiátricos como esquizofrenia, que incluyen depresión, autismo y ansiedad [7]. Además, el trastorno por déficit de atención con hiperactividad (TDAH) como al menos en un subconjunto de pacientes muestra alteraciones en el funcionamiento del eje HPA [5].

En una investigación realizada en el año 2017, anunciaron que el estrés durante el embarazo puede afectar a las generaciones posteriores, lo que se demuestra por una mayor susceptibilidad a los problemas de salud de la infancia y la edad adulta en los hijos y nietos. En un diseño de estudio transversal, una muestra de 121 jóvenes adolescentes y sus abuelas maternas, las mismas que viven en São Gonçalo, una ciudad ubicada en el estado de Río de Janeiro, Brasil, la misma que presenta una alta proporción de familias de bajos ingresos y altos niveles de violencia doméstica y comunitaria, se pudo concluir que la exposición de la abuela a la violencia interpersonal durante el embarazo se asoció con 27 sitios CpG metilados diferencialmente en los niños que se asignan a 22 genes con anotaciones únicas. Dentro de los sitios de CpG asociados con el estrés prenatal de la abuela, cinco tenían un significado de alta confianza (FDR $<0,05$ ). Los genes más metilados diferencialmente (grupo de alta confianza) asociados con el estrés de la abuela durante el embarazo fueron: CFTR y CORIN los mismo que están involucrados en los procesos del sistema circulatorio como hipertensión e insuficiencia cardíaca y en las anomalías congénitas, BARX1 y SMYD3. $B A R X 1$ se ha asociado al desarrollo embrionario y craneofacial, la odontogénesis y la organogénesis estomacal. Asimismo, la exposición prenatal al estrés, se ha asociado a la presentación de tasas elevadas de obesidad y perfil lipídico alterado [8].

Estudios previos han demostrado que la desregulación de CFTR tiene un impacto en la liberación de vitamina $\mathrm{D}$, que puede afectar los síntomas de depresión y de TEPT [9].

\subsection{Contaminación atmosférica}

La contaminación atmosférica es considerada como la mayor causa de morbimortalidad infantil. Existe escepticismo sobre los efectos tempranos sobre la salud postnatal [10].

Varios estudios ponen en evidencia los efectos asociados con la exposición prenatal a diversos compuestos como: Monóxido de nitrógeno (NO), Dióxido de nitrógeno $\left(\mathrm{NO}_{2}\right)$, Dióxido de carbono $\left(\mathrm{CO}_{2}\right)$, Monóxido de carbono (CO), Hidrocarbonos policíclicos aromáticos (HPA), Dióxido de azufre $\left(\mathrm{SO}_{2}\right)$, Mercurio $(\mathrm{Hg})$ y humo de tabaco [10].

En el sistema respiratorio, la exposición a contaminantes ambientales como el tabaco, además madres fumadoras, ha sido asociada a la aparición de asma y respiración sibilante en bebés. En cambio, en madres no fumadoras, la presencia de asma, respiración sibilante y bronquitis/bronquiolitis se ha relacionado con una exposición temprana al NO [11]. 


\subsection{Plomo}

En cuanto a los efectos más sobresalientes de la exposición al plomo en la etapa intrauterina, influyen sobre el sistema nervioso central causando una disminución del coeficiente de inteligencia, dificultades en el aprendizaje y fracasos escolares [12].

El descenso del Coeficiente Intelectual ya se observa a partir de una plumbemia de $5 \mathrm{pg} / \mathrm{dL}$. La exposición perinatal o infantil a plomo genera el desarrollo de una personalidad hiperactiva y agresiva. Además, se ha asociado un aumento de riesgo para conductas antisociales y delictivas al aumento del nivel de plomo en la tibia como reflejo de exposición prenatal ya que se incorpora al hueso prácticamente de por vida por su osificación al nacer [12].

\subsection{Ftalatos y fenoles}

En México, mediante un estudio se hizo seguimiento a 250 niños (8 a 14 años), nacidos de madres en las que se analizaron metabolitos de ftalato en orina materna y concentraciones de Bisfenol durante el primer, segundo y tercer trimestre de embarazo. Los niveles de Bisfenol y de varios metabolitos de ftalatos (monobenzil ftalato, mono- 3carboxiopropil ftalato y la suma de mono-n-butil ftalato y mono isobutil ftalato) durante el tercer trimestre, se asociaron con reducción de parámetros de adrenarquia (que influyen en la maduración testicular, aparición de vello pubiano) y con un aumento de globulina fijadora de hormonas sexuales sérica [13].

\subsection{Nutrición}

El estado nutricional en el embarazo es un factor importante debido a que es modificable y a su vez capaz de ser modificado. Varios aspectos de la dieta materna tienen gran repercusión dentro del crecimiento y el desarrollo fetal, incluyendo el consumo de frutas, vegetales, pescado, carne, leche y hierro, ácido fólico, vitamina D y otras vitaminas y antioxidantes como la vitamina $E$ [14].

El hierro, tiene efectos directos sobre el desarrollo fetal ya que una suplementación adecuada disminuye el riesgo de bajo peso en el recién nacido, efecto relacionado con enfermedades cardiovasculares a futuro en la vida adulta.

El déficit de yodo altera el desarrollo cognitivo. La vitamina A y betacarotenos son importantes en las etapas fi $\neg$ nales del embarazo debido a que ayuda al desarrollo pulmonar. La suplementación con ácido docosahexaenoico (DHA) alarga la gestación, incrementa el peso neonatal y aporta bene $\neg$ ficios en el neurodesarrollo [14].

La suplementación del ácido fólico periconcepcional tiene un claro efecto protector sobre la aparición de los defectos del tubo neural. En una investigación basada en la suplementación continua de ácido fólico en el embarazo más allá del período inicial recomendando de su ingesta, concluyó que tiene efectos beneficiosos sobre el desarrollo cognitivo infantil [15].

En cuanto a la vitamina B12, se sabe que su deficiencia está relacionada con el aumento del índice de masa corporal, y la diabetes gestacional. Un estudio realizado 
con 117 mujeres en Sumatra, sometiéndose a análisis antropométricos, bioquímicos, de ingesta dietética y pruebas genéticas, y a través de una construcción de puntuaciones de riesgo genético (GRS) basadas en nueve polimorfismos de un solo nucleótido asociados a la vitamina B12 (SNP) (B12-GRS) y nueve SNP metabólicos (GRS metabólico), se pudo conocer que si hay un riesgo asociado de padecer diabetes mellitus, especialmente a un paupérrimo consumo de fibra [16].

La absorción de nutrientes fetales juega un papel importante en el desarrollo de numerosas enfermedades. Se recomienda una dieta mediterránea para prevenir el desarrollo de alergias del niño, especialmente una alta ingesta de grasas omega-3. Los ácidos encontrados en el aceite de pescado mostraron efectos antiinflamatorios en las células mononucleares de cordón umbilical y la reducción del estrés oxidativo en la placenta, que a futuro disminuyen el riesgo de enfermedades alérgicas [17].

Aunque la vitamina $D$ se asocia positivamente con la protección de sibilancias y eczema, recientemente, se asoció los efectos positivos de la suplementación con vitamina $D$ durante el segundo y tercer trimestre del embarazo con la capacidad de respuesta en estímulos innatos y mitógenos por regulación positiva de la producción de citocinas y la expresión de TLR2 y TLR9 mostrada en las células mononucleares del cordón umbilical. Además, la producción de IL-17A aumentó con la estimulación de las células T, implicados en la defensa inmune contra los patógenos [18]

\subsection{Genética}

La investigación genómica reciente aumenta la comprensión de las causas de las enfermedades y fortalece la evidencia de que muchas enfermedades, incluso aquellas con una aparición tardía, son causadas en parte por ambientes prenatales adversos inducidos genéticamente [19].

El crecimiento fetal es un determinante importante de la salud y la enfermedad en niños y adultos. Las medidas de anormalidad del crecimiento fetal se asocian con morbilidad y mortalidad perinatal y resultados adversos para la salud a largo plazo. Las interacciones complejas entre factores genéticos y ambientales, incluidas las variaciones genéticas fetales y parentales, la nutrición materna y la función placentaria, juegan un papel importante en el crecimiento fetal [20].

Las madres determinan en gran medida el entorno fetal; por lo tanto, se espera que los genotipos maternos influyan en el desarrollo fetal. Dos tipos de interacciones gen-gen son posibles durante el embarazo: interacción intrageneracional e interacción intergeneracional. El primero se refiere a las interacciones gen-gen dentro del genoma de un individuo para afectar el resultado de su propia enfermedad, y el segundo hace alusión a los efectos intergeneracionales que conducen a entornos beneficiosos o conflictivos entre una madre y su feto. Las interacciones intergeneracionales pueden ocurrir entre genes en el genoma de un niño que afectan el fenotipo de la madre o entre genes en el genoma de una madre que afectan el fenotipo del niño. También puede haber interacciones entre los genes maternos y los genes fetales, como la incompatibilidad genotipo materno-fetal, que causan cambios en el fenotipo de la madre o del niño. Las interacciones gen-gen también se han estudiado como causas 
de complicaciones del embarazo, como la hipertensión gestacional, la diabetes, el metabolismo y la preeclampsia [19, 21].

Los efectos de las incompatibilidades del genotipo fetal materno deben ser modulados por el entorno prenatal. Como ejemplo, las infecciones maternas durante el embarazo se han implicado como factores de riesgo para trastornos en niños tan diversos como anomalías congénitas, pérdida de audición y esquizofrenia. De hecho, puede haber efectos de infección del tracto urinario materno por genoma en la esquizofrenia. Por lo tanto, es importante comprender los factores de riesgo genéticos subyacentes a la infección recurrente del tracto urinario (ITU). Zaffanello y col. resume la investigación sobre los determinantes genéticos de la infección urinaria y encuentra que, de los genes candidatos estudiados, solo HSPA1B, CXCR1, CXCR2, TLR2, TLR4 y TGF-1 están significativamente asociados con la infección urinaria recurrente. De estos genes, CXCR1 es el más ampliamente estudiado y respaldado. CXCR1 codifica el receptor para la quimiocina IL-8, y las quimiocinas son una parte importante del proceso inflamatorio $[19,22]$.

\subsection{Epigenética}

Los mecanismos epigenéticos son esenciales para la diferenciación celular y, por lo tanto, para el desarrollo. Los últimos años han generado un gran interés en el paisaje epigenético del embarazo en desarrollo, y cómo el cambio epigenético, mediado por la influencia ambiental, modifica la trayectoria de desarrollo y el fenotipo posterior [23].

El término epigenética define una variedad de procesos que causan cambios heredables mitótica y meióticamente en la expresión génica sin modificar la secuencia del ácido desoxirribonucleico (ADN); particularmente, la metilación del ADN, la modificación de histonas y el Ácido ribonucleico (ARN) no codificante, son los principales mecanismos subyacentes a las modificaciones epigenéticas, siendo estos cruciales para un desarrollo saludable feto neonatal [1].

La definición moderna de epigenética tiene en cuenta la plasticidad, las modificaciones del ADN o los factores asociados que tienen contenido de información, además de la secuencia de ADN en sí, se mantienen durante la división celular, influenciados por el entorno y causan cambios estables en la expresión génica. Por lo tanto, el paisaje epigenético ahora se ve más dinámicamente de lo que era inicialmente [3].

Antes de la implantación, el epigenoma es altamente dinámico. La reprogramación epigenética extensa ocurre dos veces durante el desarrollo de mamíferos. El primer evento de desmetilación a escala del genoma ocurre durante la generación de células germinales masculinas y femeninas, donde se produce una eliminación de las marcas epigenéticas incluidos los genes impresos de los gametos. La evidencia reciente de ratones indica que las marcas epigenéticas no se borran completamente durante la gametogénesis. Este hallazgo sugiere un potencial para la herencia epigenética transgeneracional a través de los gametos. El segundo evento de reprogramación epigenética ocurre temprano después de la fertilización, donde el óvulo fertilizado se somete a una serie de divisiones celulares antes de la implantación, llegando finalmente a la etapa de blastocisto. La metilación del ADN en el cigoto disminuye progresivamente; 
la metilación promedio en la fertilización es del $41 \%$, y esto disminuye en un $9 \%$ en la etapa de 2 células. En la etapa de blastocisto, el genoma está casi completamente hipometilado; sin embargo, las marcas epigenéticas impresas permanecen intactas durante esta etapa [23].

Las células de blastocistos totipotentes pronto proliferan y se diferencian en 2 linajes, la masa celular pluripotente interna $(\mathrm{MCl})$, que se convertirá en el feto en desarrollo, y el trofectodermo, del cual surgen los tejidos extraembrionarios. Esta diferenciación implica cambios en la expresión génica mediada por distintas alteraciones epigenéticas, a medida que se produce la primera metilación de ADN de novo y otros eventos epigenéticos. La evidencia sugiere que el restablecimiento de las marcas epigenéticas es más limitado en el trofectodermo, lo que se refleja en la hipometilación del tejido extraembrionario (es decir, la placenta) en comparación con las células somáticas [23].

En el embrión en desarrollo, los cambios sutiles en el microambiente durante etapas específicas del desarrollo impulsan el cambio epigenético asociado con la diferenciación celular. Sin embargo, esta sensibilidad inherente a la influencia externa, que es un sello distintivo del desarrollo temprano, haciendo que las marcas epigenéticas sean susceptibles a la influencia ambiental externa [23, 24].

La evidencia reciente ha demostrado que algunos de los cambios epigenéticos resultantes de la nutrición temprana y el microbioma pueden heredarse transgeneracionalmente, lo que tiene un impacto significativo en la evolución [1].

\subsection{Microbiota}

\subsubsection{Microbiota dentro del útero}

La evidencia contradice el dogma de que el feto reside en un ambiente estéril y que el recién nacido solo alcanza su microbiota después de la exposición al ambiente extrauterino. Se sabe desde hace más de 30 años que incluso sin un saco amniótico roto, el líquido amniótico con frecuencia contiene niveles significativos de bacterias. La infección intrauterina y una respuesta inflamatoria fetal posterior se han relacionado con la prematuridad, enfermedad cerebral, pulmonar y ocular después del parto prematuro. Los estudios realizados en la última década utilizando técnicas no basadas en cultivos revelan una presencia significativa de microbios en la placenta, el líquido amniótico y el meconio, los que parecen diferir dependiendo de la etapa de madurez fetal [25].

Por lo tanto, el dogma clásico de que el recién nacido emerge de un entorno estéril debe considerarse como una suposición. Está claro que el sistema inmune fetal, especialmente el del intestino fetal, está evolucionando rápidamente durante la gestación y que las morbilidades posnatales como la enterocolitis necrotizante, la enfermedad pulmonar crónica, la enfermedad de la sustancia blanca del cerebro y otras afecciones inflamatorias pueden verse afectadas por la colonización microbiana que ocurre mucho antes de que el bebé abandone el útero [25].

El embarazo es el comienzo de la exposición bacteriana para el embrión y el feto en desarrollo. Los orígenes de estas bacterias incluyen el tracto gastrointestinal materno y estas bacterias se han aislado en la sangre del cordón umbilical, la placenta del 
líquido amniótico, las membranas fetales y el meconio. Además, estas bacterias pueden contribuir a la estimulación inmunológica que puede provocar lesiones posteriores en el feto y el recién nacido o incluso la tolerancia inmunológica que conduce a la prevención de ciertas enfermedades, como las asociadas con la autoinmunidad y la alergia en la edad adulta [25].

\subsubsection{Colonización microbiana intestinal y epigenética}

El tracto gastrointestinal es el sitio más importante de las interacciones huéspedmicrobio y se ha demostrado que el establecimiento de un microbiota intestinal indígena durante la vida temprana tiene un gran impacto en la fisiología humana.

Aunque todavía existe controversia sobre el concepto de útero estéril versus el concepto de colonización en el útero es difícil discutir que el ambiente microbiano del feto en el útero tiene implicaciones importantes para la salud y la enfermedad. El establecimiento postnatal del microbiota intestinal está influenciado por varios factores: Modo de parto, contacto con la madre (como el cuidado de piel a piel), composición de la dieta y administración de agentes farmacológicos, especialmente antibióticos. Inmediatamente después del nacimiento, el microbioma intestinal infantil tiene una diversidad de especies relativamente baja y altas tasas de flujo bacteriano. A los 3 años de edad, este flujo comienza a estabilizarse. Se cree que los estafilococos, estreptococos, Escherichia coli y enterobacterias son los primeros colonizadores del intestino. Las bacterias anaerobias facultativas reemplazan posteriormente a estos taxones y consisten en grandes abundancias relativas de actinobacterias y firmicutes Esto está influenciado en gran parte por la dieta; por ejemplo, la lactancia materna parece estimular el crecimiento de las especies de bifidobacterias.

Por lo tanto, las influencias ambientales tempranas que influyen en la microbiota intestinal durante este período crucial de desarrollo pueden modificar su composición hacia perfiles más patógenos que, a su vez, pueden persistir hasta la edad adulta y ejercer efectos duraderos sobre la salud y la enfermedad [26].

Entre los posibles mecanismos a través de los cuales las bacterias intestinales pueden influir en la salud humana, prevalecen las modificaciones epigenéticas. El potencial directo de los microbios para inducir cambios epigenéticos en el huésped ha sido demostrado recientemente por la evidencia de patrones específicos de microbios de modificación del ADN epigenético después de la exposición a organismos comensales o patógenos en células humanas inmaduras de epitelios intestinales. Curiosamente, el mismo estudio también informó que la programación epigenética prenatal inducida por glucocorticoides da como resultado una composición alterada de la microbiota intestinal en ratones, lo que sugiere la existencia de interacciones complejas entre el microbioma y el epigenoma [26].

El papel de la microbiota como modulador epigenético está ganando cada vez más atención y, aunque los mecanismos subyacentes aún necesitan ser aclarados parcialmente, la evidencia actual respalda una correlación significativa entre la composición de la microbiota intestinal y los cambios en genes relevantes para el desarrollo inmunológico, metabólico y neurológico y funciones [26]. 
Cuando los recién nacidos nacen, tienen relativamente pocas bacterias intestinales. Después del nacimiento, la microbiota intestinal se expande rápidamente, progresando constantemente hacia una composición adulta. La interrupción de la flora intestinal normal (disbiosis) está asociada con muchas enfermedades humanas [26].

\section{(i) Desarrollo de la microbiota intestinal en lactantes}

Después del nacimiento, la población bacteriana intestinal del bebé se expande rápidamente. Las primeras bacterias que colonizan a los bebés normales son las especies Streptococcus, Staphylococcus, E. coli, Lactobacillus y Enterobacter. Estos colonizadores iniciales consumen oxígeno, creando así un ambiente bajo en oxígeno que selecciona el crecimiento de especies bacterianas anaerobias. Por lo tanto, la flora cambia de bacterias aerobias a bacterias anaerobias facultativas, y finalmente a anaerobios estrictos entre el día de la vida 3 y 7 . Estas bacterias anaerobias consisten principalmente en Clostridia, Bifidobacterium especies y miembros de Firmicutes phyla [26].

Varios factores, incluido el modo de parto, juegan un papel importante en la colonización inicial de los intestinos fetales. Los bebés nacidos por vía vaginal generalmente se siembran con flora vaginal materna, incluidas las especies Lactobacillus y Prevotella, mientras que los bebés nacidos por cesárea están colonizados por la flora de la piel, incluidos Staphylococcus y Corynebacterium. De hecho, el microbiota vaginal materno experimenta una disminución en su diversidad general de especies, mientras que Lactobacillus experimenta una rápida proliferación, dominando al final de la gestación, tal vez para ayudar en la transmisión vertical. Por el contrario, los recién nacidos por cesárea no solo han disminuido en gran medida las cantidades iniciales de bifidobacterias, sino también un retraso en su colonización. De 1 a 3 años, la microbiota del bebé está bien colonizado con especies similares al bioma adulto, principalmente con especies anaeróbicas, incluidos los miembros de Firmicutes y Bacteroides phyla [26, 27].

\subsection{Antibióticos}

La colonización microbiana del tracto gastrointestinal infantil (TGI) comienza al nacer, está conformada por la microbiota materna y es profundamente alterado por el tratamiento con antibióticos. El tratamiento antibiótico de las madres durante el embarazo influye en la colonización de la microbiota del TGI de sus bebés [28].

La exposición a antibióticos prenatal/postnatal y la intensidad de la exposición a antibióticos podrían afectar la composición del microbiota intestinal en los recién nacidos. Se puede suponer con seguridad que los factores ambientales como las bacterias resistentes a los antibióticos en la unidad de cuidados intensivos neonatales (UCIN) pueden tener un profundo impacto en la composición del microbiota intestinal temprana en los recién nacidos prematuros con exposición prenatal y postnatal a antibióticos. La microbiota intestinal de los recién nacidos prematuros incluye bacterias resistentes a los antibióticos en la UCIN, lo que representa una amenaza para la supervivencia de los lactantes [29, 30]. 


\section{Conclusiones}

Resulta importante destacar el gran impacto que genera la interrelación de la exposición a factores ambientales, la nutrición y la microbiota, y sus repercusiones sobre la genética y epigenética en el desarrollo feto neonatal, por lo que se considera fundamental, tener una comprensión profunda respecto al tema, orientado en la búsqueda de avances especialmente en la prevención de posibles situaciones que constituyen potenciales noxas para el nuevo ser.

La investigación sobre métodos para detectar estas interacciones y para comprender los mecanismos de estas interacciones apenas está comenzando. Sin embargo, hay evidencia de que estas interacciones juegan un papel importante en el desarrollo humano. Sin embargo, se necesita más investigación para determinar cuán grande es el impacto que tienen en la determinación de los fenotipos humanos.

\section{Agradecimiento}

Agradecemos al Seminario Internacional De Salud, Alimentación Y Nutrición Humana (SISANH 2020), de la Facultad de Salud pública de la Escuela Superior Politécnica de Chimborazo, por permitir la difusión del presente artículo de revisión y a su Comité Editorial por su ahínco colaborativo en la investigación científica.

\section{Conflictos de Interés}

Los autores declaramos no tener ningún conflicto de interés.

\section{Limitaciones de Responsabilidad}

Los autores declaramos que toda la información recopilada en el presente documento es de nuestra responsabilidad y no de la institución en la que laboramos.

\section{Fuentes de Financiación}

La financiación del presente documento proviene de los mismos autores.

\section{References}

[1] Indrio F, Martini S, Francavilla R, et al. Epigenetic matters: The link between early nutrition, microbiome, and long-term health development. Frontiers in Pediatrics. 2017;5.

[2] Tiffon $C$. The impact of nutrition and environmental epigenetics on human health and disease. Int $J$ Mol Sci. 2018;19(11).

[3] Feinberg AP. The key role of epigenetics in human disease prevention and mitigation. New England Journal of Medicine. Massachussetts Medical Society. 2018; 378:1323-34.

[4] Kundakovic M, Jaric I. The epigenetic link between prenatal adverse environments and neurodevelopmental disorders. Genes. MDPI AG. 2017;8. 
[5] Palma-Gudiel H, Córdova-Palomera A, Eixarch E, Deuschle M, Fañanás L. Maternal psychosocial stress during pregnancy alters the epigenetic signature of the glucocorticoid receptor gene promoter in their offspring: A meta-analysis. Epigenetics. 2015;10:893-902.

[6] Bedregal P, Shand B, Santos MJ, Ventura-Juncá P. Aportes de la epigenética en la comprensión del desarrollo del ser humano. Revista Medica de Chile. 2010;138:366-72.

[7] Cáceres R, Martínez-Aguayo JC, Arancibia M, Sepúlveda E. Efectos neurobiológicos del estrés prenatal sobre el nuevo ser. Revista Chilena de Neuro-Psiquiatria. 2017;55:103-13.

[8] Serpeloni F, Radtke K, de Assis SG, Henning F, Nätt D, Elbert T. Grandmaternal stress during pregnancy and DNA methylation of the third generation: An epigenome-wide association study. Transl Psychiatry. 2017;7(8):e1202.

[9] Hoang MT, DeFina LF, Willis BL, Leonard DS, Weiner MF, Brown ES. Association between low serum 25hydroxyvitamin $\mathrm{D}$ and depression in a large sample of healthy adults: The Cooper Center longitudinal study. Mayo Clin Proc. 2011;86(11):1050-5.

[10] Toranõ EG, Garciá MG, Fernández-Morera JL, Ninõ-Garciá P, Fernández AF. The impact of external factors on the epigenome: In utero and over lifetime. BioMed Research International. 2016.

[11] Vieira SE. The health burden of pollution: The impact of prenatal exposure to air pollutants. Int J COPD. 2015 Jun;10:1111-21.

[12] Tarr H, Raymond RE, Tufts M. The effects of lead exposure on school outcome among children living and attending Public Schools in Detroit, MI. Partners in Good Health.

[13] Watkins DJ, Sánchez BN, Téllez-Rojo MM, et al. Impact of phthalate and BPA exposure during in utero windows of susceptibility on reproductive hormones and sexual maturation in peripubertal males. Environ Heal A Glob Access Sci Source. 2017;16(1).

[14] Pullar J, Wickramasinghe K, Demaio AR, et al. The impact of maternal nutrition on offspring's risk of non-communicable diseases in adulthood: A systematic review. J Glob Health. 2019;9(2).

[15] McNulty H, Rollins M, Cassidy T, et al. Effect of continued folic acid supplementation beyond the first trimester of pregnancy on cognitive performance in the child: A follow-up study from a randomized controlled trial (FASSTT Offspring Trial). BMC Med. 2019;17(1).

[16] Surendran S, Aji AS, Ariyasra U, et al. A nutrigenetic approach for investigating the relationship between vitamin B12 status and metabolic traits in Indonesian women. J Diabetes Metab Disord. 2019;

[17] Nowak E, Neuner A, Landgraf-Rauf K, Schaub B. Asthma und allergieprävention. Pädiatrie up2date. 2017;12(02):143-59.

[18] Hornsby E, Pfeffer PE, Laranjo N, et al. Vitamin D supplementation during pregnancy: Effect on the neonatal immune system in a randomized controlled trial. J Allergy Clin Immunol. 2018;141(1):269-278.e1.

[19] Fu WJ, Sinsheimer JS, Elston RC. Gene-gene interaction in maternal and perinatal research. Journal of Biomedicine and Biotechnology. 2010.

[20] Workalemahu T, Grantz KL, Grewal J, Zhang C, Louis GMB, Tekola-Ayele F. Genetic and environmenta influences on fetal growth vary during sensitive periods in pregnancy. Sci Rep. 2018;8(1).

[21] Traglia M, Croen LA, Jones KL, et al. Cross-genetic determination of maternal and neonatal immune mediators during pregnancy. Genome Med. 2018;10(1).

[22] Lunde A, Melve KK, Gjessing HK, Skjaerven R, Irgens LM. Genetic and environmental influences on birth weight, birth length, head circumference, and gestational age by use of population-based parentoffspring data. Am J Epidemiol. 2007;165(7):734-41.

[23] Januar V, Desoye G, Novakovic B, Cvitic S, Saffery R. Epigenetic regulation of human placental function and pregnancy outcome: Considerations for causal inference. American Journal of Obstetrics and Gynecology. 2015;213:S182-96.

[24] Teh AL, Pan H, Chen L, et al. The effect of genotype and in utero environment on interindividual variation in neonate DNA methylomes. Genome Res. 2014;24(7):1064-74.

[25] Neu J. Developmental aspects of maternal-fetal, and infant gut microbiota and implications for long-term health. Matern Heal Neonatol Perinatol. 2015;1(1).

[26] Elgin TG, Kern SL, McElroy SJ. Development of the neonatal intestinal microbiome and its association with necrotizing enterocolitis. Clinical Therapeutics. 2016;38:706-15.

[27] Henderickx JGE, Zwittink RD, Van Lingen RA, Knol J, Belzer C. The preterm gut microbiota: An inconspicuous challenge in nutritional neonatal care. Frontiers in Cellular and Infection Microbiology. 2019;9.

[28] Tekieli L-S, Radens CM, Williams BL, N Gabriela Gonzalez-Perez ES, Hicks AL. Microbiome and antiviral immunity development of the neonatal intestinal maternal antibiotic treatment impacts. J Inmunol. 2016;196:3768-79.

[29] Zou Z-H, Liu D, Li H-D, et al. Prenatal and postnatal antibiotic exposure influences the gut microbiota of preterm infants in neonatal intensive care units. Ann Clin Microbiol Antimicrob. 2018;17(1):9.

[30] Tirone C, Pezza L, Paladini A, et al. Gut and lung microbiota in preterm infants: Immunological modulation and implication in neonatal outcomes. Frontiers in Immunology. 2019;10:2910. 